\title{
APORTE DE NUTRIENTES E PRODUTIVIDADE DE ESPÉCIES ARBÓREAS E DE CULTIVOS AGRÍCOLAS EM SISTEMAS AGROFLORESTAIS NA AMAZÔNIA
}

\author{
Marcelo Francia Arco-Verde ${ }^{1}$, Ivan Crespo Silva ${ }^{2}$, Moisés Mourão Júnior ${ }^{3}$ \\ ${ }^{1}$ Eng. Florestal, Dr., EMBRAPA Roraima, Boa Vista, RR, Brasil - arcoverd @ig.com.br \\ ${ }^{2}$ Eng. Florestal, Dr., Depto. de Ciências Florestais, UFPR, Curitiba, PR, Brasil - ivancrespo@ufpr.br \\ ${ }^{3}$ Biólogo, M.Sc., EMBRAPA Roraima, Boa Vista, RR, Brasil - mmourao@cpafrr.embrapa.br
}

Recebido para publicação: 26/09/2007 - Aceito para publicação: 04/08/2008

\begin{abstract}
Resumo
Combinar espécies compatíveis e adequadas a uma ou mais funções, selecionar tecnologias apropriadas às condições ambientais e sociais e desenhar e planejar modalidades específicas de multicultivo para satisfazer determinada demanda de produção são algumas das tarefas necessárias para se alcançarem as metas dos sistemas agroflorestais produtivos. Neste trabalho, o principal objetivo foi avaliar o crescimento e a produtividade de espécies arbóreas e agrícolas em sistemas agroflorestais, com diferentes práticas de manejo, implantados no estado de Roraima. O estudo foi conduzido em campo experimental no estado de Roraima, com informações geradas entre 1995 e 2002. Dois modelos agroflorestais foram estudados, sendo compostos por culturas anuais no início da implantação seguidas de bananeira (Musa sp. cv. Missouri), ingá-de-metro (Inga edulis), gliricídia (Gliricidia sepium), cupuaçuzeiro (Theobroma grandiflorum), pupunheira (Bactris gasipaes), castanha-do-brasil (Bertholletia excelsa) e cupiúba (Goupia glabra). A baixa fertilidade dos solos prejudicou o crescimento e a produtividade das culturas anuais, sendo que a mandioca foi menos afetada. Os nutrientes contidos na biomassa provenientes das podas de ingá-de-metro e gliricídia contribuíram para a manutenção da fertilidade do solo. O cupuaçuzeiro foi a espécie que apresentou melhor produtividade em solos com maior fertilidade, tornando-se o componente mais importante dos SAFs avaliados.

Palavras-chave: Biomassa; espécies anuais; fruteiras; crescimento; Amazônia.
\end{abstract}

\begin{abstract}
Nutrient reliese and productivity of agroforestry species in agroforestry systems in Amazônia. To join together trees with technologies, to select appropriate technologies with different categories of users of the land, and design a specific technology to offer an effective solution for one problem of use of the land, are some of the tasks necessary to reach the goals of the productive agroforestry systems. The main objective of this work was to evaluate the growth and the productivity of agroforestry systems, with different practical of handling, implanted in the state of Roraima. The study was lead in experimental field in the state of Roraima, with information generated between 1995 and 2002. Two agroforestry models were studied, being composites for annual cultures at the beginning of the implantation followed of banana tree (Musa sp. cv. Missouri); ingá-de-metro (Inga edulis); gliricídia (Gliricidia sepium); cupuassu (Theobroma grandiflorum); palm heart (Bactris gasipaes); Brazil nut (Bertholletia excelsa); cupiuba (Goupia glabra). The low fertility of soil affected the growth and the productivity of the annual crops, were the cassava had the best results. The biomass from the pruning of ingá-of-meter and gliricídia contributed for the maintenance of the soil fertility. Cupuassu was the specie that presented better productive, becoming the component most important of the agroforestry systems.
\end{abstract}

Keywords: Biomass; annual crops; fruits; growth; Amazonia.

\section{INTRODUÇÃO}

Os sistemas agroflorestais (SAFs) são uma opção viável entre os sistemas de produção sustentáveis existentes, com o principal objetivo de contribuir para o bem-estar nutricional, social e 
econômico dos produtores rurais, particularmente aqueles de baixa renda, assim como para a conservação dos recursos naturais.

No estado de Roraima, os trabalhos de pesquisa, com enfoque em SAFs, iniciaram em 1995, com uma equipe multidisciplinar da Embrapa buscando oferecer modelos de SAFs produtivos e sustentáveis para os produtores rurais da região.

De acordo com as necessidades identificadas, foram desenhados modelos agroflorestais considerando-se fatores fundamentais para obter níveis adequados de crescimento e produtividade: i) seleção da área do estudo, que deveria ter características semelhantes às dos produtores rurais; ii) identificação das espécies nativas e introduzidas e suas funções em cada modelo; iii) definição da dinâmica dos componentes, ou seja, estabelecer a ordem de implantação de cada espécie, assim como o momento para substituição ou retirada; iv) definição das práticas silviculturais e de manejo para cada espécie; v) identificação das atividades e custos de mão-de-obra nas diferentes fases de implantação dos sistemas; vi) estimativa do crescimento e da produtividade de cada componente ao longo do tempo, caracterizando os diferentes benefícios e, consequentemente, fontes de recursos financeiros dos SAFs a cada ano; e vii) definição do planejamento para incentivar os produtores rurais a adotarem os SAFs em suas propriedades.

Durante todo o processo de consolidação dos SAFs no referido estado, sempre houve a necessidade de obterem-se informações técnicas sobre as diferentes etapas de implantação e manejo dos modelos agroflorestais, de forma a poderem ser apresentadas aos representantes do meio rural informações técnicas e biofísicas de diferentes modelos agroflorestais para o estado de Roraima.

O potencial dos SAFs é, há alguns anos, reconhecido na região, porém os modelos usados precisam de validação junto aos produtores, em termos de produção e sustentabilidade. É necessário, portanto, transformar esse potencial produtivo e de serviços em fato compatível com a realidade rural e com a perspectiva desejável de uma atividade sustentável do uso da terra. Combinar espécies compatíveis e adequadas a uma ou mais funções, selecionar tecnologias apropriadas às condições ambientais e sociais e desenhar e planejar modalidades específicas de multicultivo para satisfazer determinada demanda de produção são algumas das tarefas necessárias para se alcançarem as metas dos sistemas agroflorestais produtivos.

O objetivo deste trabalho foi avaliar o crescimento e a produtividade de diferentes espécies arbóreas e de cultivos agrícolas usados como componentes de sistemas agroflorestais, submetidos a diferentes práticas de manejo.

\section{REVISÃO DE LITERATURA}

Na Amazônia, a estratégia para a produção agropecuária extensiva se baseia em duas vertentes características, que são a produção agrícola em monocultivo e a pecuária a pleno sol, geralmente com subutilização dos recursos disponíveis. Com a necessidade de melhorar o uso da terra devido ao aumento populacional e à crescente demanda por bens e serviços agroflorestais, políticas públicas têm voltado sua atenção para propostas que integrem cultivos anuais, espécies forrageiras e madeiráveis em uma mesma unidade de área nos trópicos úmidos (NAIR, 1993).

A conversão de sistemas agrícolas convencionais em sistemas agroflorestais aumenta a estabilidade dos agroecossistemas, protege-os de alterações ambientais, melhora as propriedades químicas e físicas do solo e reduz o risco de erosão devido, principalmente, à formação da liteira e ao aumento da matéria orgânica. Soma-se a isso a demanda por benefícios econômicos, por segurança alimentar e por um desenvolvimento sustentável, que propiciam novas oportunidades para agricultores e empresários envolvidos nesse processo (KRISHNAMURTHY; ÁVILA, 1999).

Nos sistemas agroflorestais, o ciclo da matéria orgânica geralmente ocorre a partir da produção de resíduos vegetais que se incorporam ao solo, caindo primeiro sobre a liteira, onde serão decompostos e incorporados em função dos processos de mineralização e humificação (FASSBENDER, 1993). A disponibilidade de nutrientes na fração orgânica é muito variável e a disponibilização não é imediata, já que requer mineralização prévia. A liberação lenta e progressiva é uma garantia de que os elementos minerais móveis no solo, como o nitrogênio, permaneçam retidos e não sejam facilmente perdidos por lixiviação (KASS, 1996).

Segundo Mafongoya; Giller; Palm (1998) e Fassbender; Bornemisza (1987), a composição bioquímica dos restos vegetais é muito variável, dependendo da idade e da função do órgão analisado. Os 
tecidos verdes são mais ricos em carboidratos e proteínas, e os tecidos lenhosos apresentam maiores conteúdos de compostos fenólicos (lignina) e celulose. Compostos orgânicos têm, geralmente, baixos conteúdos de nitrogênio e altos conteúdos de lignina quando comparados com a biomassa verde. Os conteúdos de polifenóis são menos previsíveis e podem aumentar ou diminuir de acordo com a idade, dependendo da espécie (MAFONGOYA, 1995).

$\mathrm{O}$ rendimento dos sistemas agroflorestais pode ser considerado como o resultado das diversas interações que ocorrem de forma direta ou indireta entre os componentes de produção. Assim, as interações entre os componentes arbóreos e não-arbóreos podem ser complementares, com ganho de rendimento; neutras, sem mudanças de rendimento; ou competitivas, com redução de rendimento, comparadas com os rendimentos dos componentes cultivados de forma isolada em uma área equivalente. $\mathrm{Na}$ prática, tanto as interações complementares como as competitivas podem ocorrer simultaneamente (KRISHNAMURTHY; ÁVILA, 1999).

\section{MATERIAL E MÉTODOS}

O estudo foi conduzido no campo experimental Confiança, pertencente à Embrapa Roraima, localizado entre as coordenadas $02^{\circ} 15^{\prime} 00^{\prime \prime} \mathrm{N}$ e 60 $39^{\circ} 54^{\prime \prime} \mathrm{W}$, no município do Cantá, a $90 \mathrm{~km}$ de Boa Vista, estado de Roraima. Essa área apresenta vegetação de floresta e clima Ami (Köppen), caracterizado como tropical chuvoso, com nítida estação seca, e amplitude térmica inferior a $5{ }^{\circ} \mathrm{C}$ entre as médias do mês mais quente e do mês mais frio.

A precipitação pluvial apresenta valores entre 1.795 e $2.385 \mathrm{~mm} \cdot \mathrm{ano}^{-1}$. Os meses mais chuvosos são maio, junho e julho, assinalando mais de $55 \%$ do total de precipitação, sendo que maio é o mês de maior precipitação (292-552 mm.mês ${ }^{-1}$ ) (MOURÃO JÚNIOR et al., 2003).

O solo é classificado como do tipo Argissolo, constituído por material mineral que tem como características argila de atividade baixa e horizonte B textural (EMBRAPA, 1999). Na tabela 1 são apresentadas as características químicas do solo no início do estudo, em 1995.

Tabela 1. Características químicas do solo no início da implantação dos SAFs.

Table 1. Chemical characteristics of soil during agroforestry establishment.

\begin{tabular}{lc}
\hline Parâmetros & Valores \\
\hline PH & 4.5 \\
Matéria orgânica $\left(\mathrm{g}^{-\mathrm{dm}^{-3}}\right)$ & 29.91 \\
Fósforo $\left(\mathrm{mg} \cdot \mathrm{dm}^{-3}\right)$ & 2.56 \\
Potássio $\left(\mathrm{mg} \cdot \mathrm{dm}^{-3}\right)$ & 40.25 \\
Cálcio $\left(\mathrm{cmolc} . \mathrm{dm}^{-3}\right)$ & 0.53 \\
Magnésio $\left(\mathrm{cmolc}_{\mathrm{dm}} \mathrm{dm}^{-3}\right)$ & 0.15 \\
\hline Fonte: autores &
\end{tabular}

A implantação dos sistemas agroflorestais iniciou em 1995, com o preparo da área consistindo de derrubada de uma capoeira de quatro anos, sem o uso de queimada da vegetação. Os dois modelos agroflorestais estudados, chamados de $\mathrm{M}_{1}$ e $\mathrm{M}_{2}$, têm na sua composição as mesmas espécies de maior ciclo biológico, ou seja:

- bananeira (Musa sp. cv. Missouri);

- ingá-de-metro (Inga edulis);

- gliricídia (Gliricidia sepium);

- cupuaçuzeiro (Theobroma grandiflorum);

- $\quad$ pupunheira (Bactris gasipaes);

- castanha-do-brasil (Bertholletia excelsa);

- cupiúba (Goupia glabra).

A diferença entre os modelos $\mathrm{M}_{1}$ e $\mathrm{M}_{2}$ está no preparo e correção do solo. No sistema $\mathrm{M}_{2}$, a área foi gradeada e o solo teve sua acidez corrigida, recebendo calagem na proporção de 2 ton.ha ${ }^{-1}$ (PRNT $100 \%$ ), sendo fertilizada com a aplicação de $40 \mathrm{~kg} \cdot \mathrm{ha}^{-1}$ de $\mathrm{P}_{2} \mathrm{O}_{5}$ e $50 \mathrm{~kg} \cdot \mathrm{ha}^{-1}$ de micronutrientes FTE BR12 no primeiro ano da implantação do estudo. No sistema $\mathrm{M}_{1}$, o solo não sofreu correção e não foi 
gradeado nem fertilizado. O espaçamento geral adotado em ambos os sistemas foi de $3 \times 2 \mathrm{~m}$, com delineamento experimental de blocos casualizados, com três repetições.

Na figura 1 pode-se observar a composição e a distribuição das espécies dos $\mathrm{SAFs}_{\mathrm{M}_{1}}$ e $\mathrm{M}_{2} \mathrm{em}$ sua fase de implantação. A ilustração representa um módulo padrão $\left(486 \mathrm{~m}^{2}\right)$ que pode ser replicado até alcançar a área desejada. Nos dois modelos, a bananeira permaneceu nos sistemas até o sexto ano, e a partir do oitavo ano ocorreu a supressão natural dos ingazeiros, plantados para adubação verde.

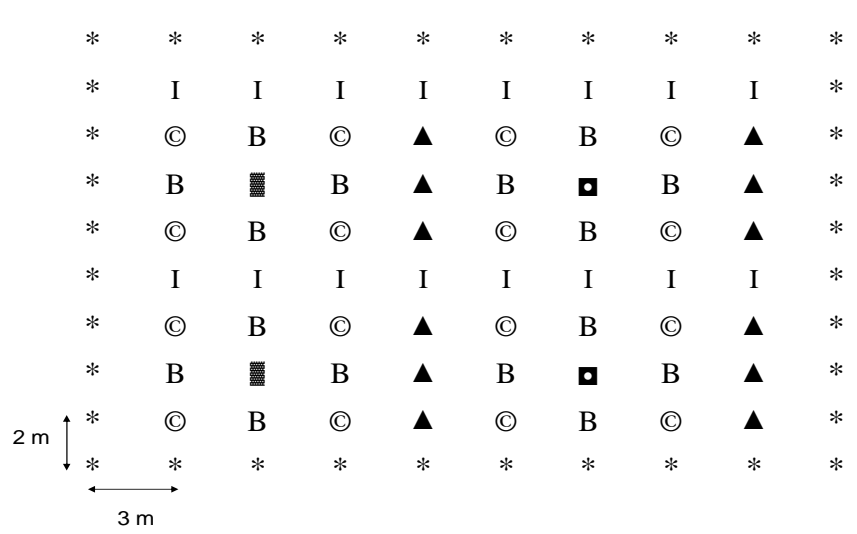

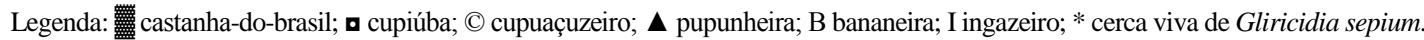
Fonte: autores.

Figura 1. Composição dos sistemas agroflorestais $\mathbf{M}_{1}$ e $\mathbf{M}_{2}$ na fase inicial.

Figure 1. Composition of agroforestry $\mathrm{M}_{1}$ and $\mathrm{M}_{2}$ models.

As duas espécies selecionadas para aumentar a fertilidade do solo e a ciclagem de nutrientes dos SAFs, Inga edulis e Gliricidia sepium, foram podadas anualmente, e a biomassa foi distribuída nas linhas de plantio. Esse procedimento favorece as demais espécies do sistema, mantendo a umidade do solo por mais tempo, diminuindo o aparecimento das plantas oportunistas e melhorando a fertilidade do solo.

Os modelos $\mathrm{M}_{1}$ e $\mathrm{M}_{2}$ apresentaram diferenças de composição em relação às espécies anuais. No modelo $\mathrm{M}_{1}$, o arroz foi cultivado no primeiro e no segundo ano, e a mandioca somente no terceiro ano.

As culturas anuais (arroz, milho, soja e mandioca) foram consideradas para a segurança alimentar dos agricultores (autoconsumo). Os cultivos anuais (ou "lavoura branca") foram implantados em sistema de plantio direto, sem gradagem, com fertilização localizada em cada cova. No modelo $\mathbf{M}_{2}$, onde o solo foi gradeado e melhor fertilizado, houve a rotação de culturas, na qual o milho foi cultivado no primeiro ano, a soja no segundo e a mandioca no terceiro (Tabela 2).

Tabela 2. Presença dos componentes agroflorestais ao longo do tempo.

Table 2. Presence of agroforestry species during the time.

\begin{tabular}{|c|c|c|c|c|c|c|c|c|c|c|c|c|c|c|c|c|c|c|c|c|}
\hline \multirow{2}{*}{$\begin{array}{l}\text { Componentes } \\
\text { dos sistemas }\end{array}$} & \multicolumn{20}{|c|}{ Anos } \\
\hline & 1 & 2 & 3 & 4 & 5 & 6 & 7 & 8 & 9 & 10 & 11 & 12 & 13 & 14 & 15 & 16 & 17 & 18 & 19 & 20 \\
\hline Arroz $^{1}$ & $\bullet$ & $\bullet$ & & & & & & & & & & & & & & & & & & \\
\hline Milho $^{2}$ & $\bullet$ & & & & & & & & & & & & & & & & & & & \\
\hline Soja $^{2}$ & & $\bullet$ & & & & & & & & & & & & & & & & & & \\
\hline Mandioca & & & $\bullet$ & & & & & & & & & & & & & & & & & \\
\hline Bananeira & & $\bullet$ & $\bullet$ & $\bullet$ & $\bullet$ & $\bullet$ & & & & & & & & & & & & & & \\
\hline Ingazeiro & 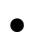 & & $\bullet$ & $\bullet$ & $\bullet$ & $\bullet$ & $\bullet$ & & & & & & & & & & & & & \\
\hline Gliricídia $^{3}$ & $\bullet$ & $\bullet$ & $\bullet$ & $\bullet$ & $\bullet$ & $\bullet$ & $\bullet$ & $\bullet$ & $\bullet$ & $\bullet$ & $\bullet$ & $\bullet$ & $\bullet$ & $\bullet$ & $\bullet$ & $\bullet$ & $\bullet$ & $\bullet$ & $\bullet$ & $\bullet$ \\
\hline Cupuaçuzeiro & $\bullet$ & $\bullet$ & $\bullet$ & $\bullet$ & $\bullet$ & $\bullet$ & $\bullet$ & $\bullet$ & $\bullet$ & $\bullet$ & $\bullet$ & $\bullet$ & $\bullet$ & $\bullet$ & $\bullet$ & $\bullet$ & $\bullet$ & $\bullet$ & $\bullet$ & $\bullet$ \\
\hline Pupunheira & & & $\bullet$ & $\bullet$ & $\bullet$ & $\bullet$ & $\bullet$ & $\bullet$ & $\bullet$ & $\bullet$ & $\bullet$ & $\bullet$ & $\bullet$ & & $\bullet$ & $\bullet$ & $\bullet$ & & $\bullet$ & $\bullet$ \\
\hline Cupiúba & & $\bullet$ & $\bullet$ & $\bullet$ & $\bullet$ & $\bullet$ & $\bullet$ & $\bullet$ & $\bullet$ & $\bullet$ & $\bullet$ & $\bullet$ & $\bullet$ & $\bullet$ & $\bullet$ & $\bullet$ & $\bullet$ & $\bullet$ & $\bullet$ & $\bullet$ \\
\hline Castanheira & & $\bullet$ & $\bullet$ & $\bullet$ & $\bullet$ & $\bullet$ & $\bullet$ & $\bullet$ & $\bullet$ & $\bullet$ & $\bullet$ & $\bullet$ & $\bullet$ & $\bullet$ & • & $\bullet$ & $\bullet$ & $\bullet$ & $\bullet$ & $\bullet$ \\
\hline
\end{tabular}

1: espécie plantada somente no modelo 1; 2: espécies plantadas somente no modelo 2; 3: espécie plantada como cerca viva. Fonte: autores. 
O experimento utilizou delineamento estatístico de blocos ao acaso com três repetições. Cada parcela experimental ocupou uma área de $2.304 \mathrm{~m}^{2}(48 \times 48 \mathrm{~m})$.

$\mathrm{O}$ arroz, plantado no sistema $\mathrm{M}_{1}$, recebeu fertilização de $200 \mathrm{~kg} \cdot \mathrm{ha}^{-1}$ de N-P-K na formulação 4$28-20+Z n$ e $100 \mathrm{~kg}^{2} \mathrm{ha}^{-1}$ de uréia no primeiro ano de plantio. No segundo ano, a fertilização de manutenção recebeu $2 \mathrm{~g}$ de N-P-K na formulação de 10-26-26 (equivalente a $80 \mathrm{~kg}$.ha ${ }^{-1}$ ) $+1 \mathrm{~g}$ de uréia nas covas (equivalente a $40 \mathrm{~kg} \cdot \mathrm{ha}^{-1}$ ).

No modelo $\mathrm{M}_{2}$, o milho foi fertilizado com $300 \mathrm{~kg} \cdot \mathrm{ha}^{-1}$ de N-P-K na formulação 4-28-20 + Zn e $200 \mathrm{~kg} \cdot \mathrm{ha}^{-1}$ de uréia no primeiro ano de implantação dos sistemas. Já a soja, plantada no segundo ano, recebeu fertilização de $3 \mathrm{~g}$ de N-P-K na formulação 10-26-26 nas covas, correspondendo a $120 \mathrm{~kg}$.ha ${ }^{-1}$. A fertilização aplicada nas culturas anuais foi calculada de acordo com as necessidades para cada cultura, nas condições edafoclimáticas semelhantes às do estudo.

A bananeira recebeu fertilização complementar de 500 g.planta $^{-1}$ de N-P-K na formulação 10-2626, no terceiro ano de implantação dos SAFs, segundo ano de plantio da cultura, nas parcelas dos modelos M1 e M2. No quarto ano, houve uma fertilização complementar, em que cada planta recebeu mais $210 \mathrm{~g}$ de sulfato de amônio, $900 \mathrm{~g}$ de superfosfato simples e $300 \mathrm{~g}$ de cloreto de potássio.

As avaliações da decomposição de folhas de castanheira (CAS), cupuaçuzeiro (CUP) e gliricídia (GLI) foram realizadas aos 15, 30, 45, 60 e 75 dias após a deposição da biomassa. A biomassa das três espécies foi acondicionada em bolsas de polietileno com malha de $1 \mathrm{~mm}$ e medindo $40 \times 40 \mathrm{~cm}$. A quantidade de material fresco (folhas) foi equivalente a $80 \mathrm{~g}$ de matéria seca para cada espécie, sendo utilizadas 60 bolsas em cada parcela, totalizando 180 bolsas distribuídas na área do estudo.

O material coletado foi limpo manualmente para a remoção de raízes e material do solo, secado em estufa com ventilação forçada a $65^{\circ} \mathrm{C}$, até atingir peso constante, e encaminhado para análise no laboratório da Embrapa Roraima. A análise dos macronutrientes foi realizada com o uso do método da digestão sulfúrica, a qual consistiu na adição prévia de $\mathrm{H}_{2} \mathrm{O}_{2}$, com pré-digestão a temperaturas entre 180 e $190{ }^{\circ} \mathrm{C}$. Essa oxidação parcial de compostos orgânicos evita a formação de espuma e frequente perda de material após a adição de $\mathrm{H}_{2} \mathrm{SO}_{4}$, no início do aquecimento (TEDESCO; WOLKWEISS; BOHNEN, 1985).

Para a determinação do nitrogênio, utilizou-se uma alíquota de $20 \mathrm{ml}$ do extrato que foi destilado em um microdestilador, após a adição de $\mathrm{NaOH}$. A seguir, o destilado foi acondicionado e avaliado em um indicador de ácido bórico, sendo titulado com $\mathrm{H}_{2} \mathrm{SO}_{4}$ diluído. $\mathrm{O}$ fósforo foi determinado por espectrofotometria e o potássio por fotometria por chama. O cálcio e o magnésio foram determinados por espectrofotometria de absorção atômica (TEDESCO, WOLKWEISS e BOHNEN, 1985).

Para as culturas da castanheira, cupuaçuzeiro e gliricídia, os valores de fitomassa foram tomados como taxas de decomposição, após ser determinada a diferença entre os respectivos valores iniciais. Esses valores foram ajustados segundo um modelo não linear do tipo sigmoidal, tendo como critério de aderência o coeficiente de determinação ajustado $\left(R_{\mathrm{aj} .}^{2}\right)$.

$$
\mathrm{y}=\beta_{0}+\left\{\beta_{1} /\left[1+\exp -\left(\mathrm{t}-\mathrm{t}_{0} / \beta_{2}\right)\right]\right\}
$$

Onde: $\mathrm{y}=$ taxa de decomposição; $\beta_{\mathrm{i}}, \mathrm{t}_{0}=$ coeficientes de modelo; $\mathrm{t}=$ tempo de avaliação.

Os valores finais de taxa de decomposição foram avaliados segundo um modelo linear geral com o uso de ANOVA de fator único e testado por meio do teste de F. Os dados foram tabulados e gerenciados na planilha eletrônica Excel 2003 versão 7. As análises foram conduzidas com o auxílio da proc nlin e da proc glm do SAS System, sendo os gráficos confeccionados com auxílio do pacote estatístico STATISTICA 5.5.

\section{RESULTADOS E DISCUSSÃO}

\section{Produção de biomassa e conteúdo de nutrientes}

Na tabela 3 são apresentados os dados de produtividade e conteúdo de nutrientes relativos às podas dos ingazeiros, realizadas nos sistemas agroflorestais no terceiro ano após a implantação.

Todos os resultados referentes à produtividade de matéria verde (M.V.) e de matéria seca (M.S.) e do conteúdo de $\mathrm{N}, \mathrm{P}, \mathrm{K}, \mathrm{Ca}$ e $\mathrm{Mg}$ foram superiores no modelo $\mathrm{M}_{2}$ quando comparados com o modelo $M_{1}$. A proporção de M.S. em relação a M.V. foi de $46 \%$, sendo de $43 \%$ nas folhas e de $50 \%$ nos ramos do ingazeiro. No terceiro ano de idade, o regime de podas dos ingazeiros no sistema $\mathrm{M}_{2}$ produziu 7.254,4 kg.ha ${ }^{-1}$ de M.V., o equivalente a 3.184,6 kg.ha ${ }^{-1}$ de M.S., quantidade $59 \%$ superior ao modelo $\mathrm{M}_{1}$. Tal 
diferença se deve, provavelmente, ao efeito da fertilização (P, FTE e calagem) e da gradagem realizadas, nas parcelas do sistema $\mathrm{M}_{2}$, sobre a produção de matéria seca dos ingazeiros (Tabela 3).

Tabela 3. Biomassa e nutrientes de Inga edulis adicionados aos SAFs aos 3 anos de idade.

Table 3. Biomass and nutrient of Inga edulis added at 3 years old.

\begin{tabular}{lcccccccc}
\hline \multirow{2}{*}{ Sistema } & \multirow{2}{*}{ Biomassa } & \multicolumn{9}{c}{ Produtividade (kg.ha $^{\mathbf{- 1}}$ ) } \\
\cline { 3 - 9 } & & M.V. & M.S. & N & P & K & Ca & Mg \\
\hline$M_{1}$ & Folha & $2.307,3$ & 1.043 & 22.79 & 1.09 & 2.13 & 7.28 & 0.86 \\
& Ramo & $1.833,4$ & 956,8 & 6.84 & 0.41 & 1.42 & 4.06 & 0.24 \\
& Total & $4.140,7$ & $1.999,7$ & 29.63 & 1.49 & 3.55 & 11.34 & 1.1 \\
$\mathrm{M}_{2}$ & Folha & 4.191 & $1.728,4$ & 43.05 & 2.32 & 2.49 & 19.92 & 3.07 \\
& Ramo & $3.063,4$ & $1.456,3$ & 9.2 & 1.32 & 1.55 & 7.41 & 0.79 \\
& Total & $7.254,4$ & $3.184,6$ & 52.25 & 3.64 & 4.03 & 27.33 & 3.87 \\
\hline
\end{tabular}

M.V.: matéria verde; M.S.: matéria seca.

Fonte: autores.

Observando-se os macronutrientes presentes na biomassa do modelo $\mathrm{M}_{2}$, constatou-se que houve maior quantidade de $\mathrm{N}$ e Ca, com valores de $52.25 \mathrm{~kg}^{-h^{-1}}$ e $27.33 \mathrm{~kg} \cdot \mathrm{ha}^{-1}$, respectivamente. Os macronutrientes estão distribuídos de forma diferenciada entre as folhas e os ramos, com maior concentração de nutrientes nas folhas, com valores oscilando entre 60 a $80 \%$ do total. Os nutrientes com maior quantidade alocada nas folhas dos modelos agroflorestais foram $\mathrm{N}, \mathrm{Ca}$ e $\mathrm{Mg}$.

\section{Decomposição da biomassa produzida}

Observa-se na tabela 4 a taxa de decomposição da biomassa produzida pela castanheira, pelo cupuaçuzeiro e pela gliricídia, no período de 75 dias. A castanheira apresentou uma taxa de decomposição de $52.9 \%$ nos primeiros 15 dias, alcançando $66.2 \%$ aos 75 dias. No cupuaçuzeiro, a taxa de decomposição foi semelhante à da castanheira, com $55.5 \%$ da fitomassa decomposta na primeira quinzena, mantendo a decomposição praticamente constante durante a avaliação. A decomposição da biomassa das folhas de gliricídia apresentou um índice de $86.8 \%$ em relação à biomassa inicial, nos primeiros 15 dias, valor superior aos encontrados com as folhas de castanheira e cupuaçuzeiro. Ressalte-se, ainda, que aos 30 dias de avaliação, praticamente toda a biomassa foliar de gliricídia havia sido decomposta, fato que demonstra o potencial da espécie para uso em SAFs como fonte de nutrientes.

Tabela 4. Taxa de decomposição (\%) da fitomassa da castanheira, cupuaçuzeiro e gliricídia.

Table 4. Decomposition rate (\%) of Brazil nut, cupuassu and gliricidia.

\begin{tabular}{|c|c|c|c|c|c|c|c|}
\hline \multirow{2}{*}{$\begin{array}{l}\text { Tempo } \\
\text { (dias) }\end{array}$} & \multicolumn{4}{|c|}{ Taxa de decomposição (\%) } & \multicolumn{3}{|c|}{ Modelo sigmoidal } \\
\hline & CAS & CUP & GLI & Coeficientes & CAS & CUP & GLI \\
\hline 0 & - & - & - & $\beta_{0}$ & $-1,074$ & $-0,846$ & $-9,062$ \\
\hline 15 & 52,9 & 55,5 & 86,8 & $\beta_{1}$ & 1,670 & 0,686 & 10,017 \\
\hline 30 & 57,0 & 54,5 & 92,1 & $\beta_{2}$ & 5,891 & 1,401 & 6,137 \\
\hline 45 & 63,5 & 55,7 & 94,7 & $\mathrm{t}_{0}$ & $-3,478$ & 2,651 & $-13,816$ \\
\hline 60 & 50,6 & 44,7 & 96,7 & $\mathrm{R}_{\mathrm{aj} .}^{2}$ & 0,88 & 0,90 & 0,99 \\
\hline 75 & 66,2 & 54,6 & 96,9 & & & & \\
\hline
\end{tabular}

CAS: castanheira; CUP: cupuaçuzeiro; GLI: gliricídia.

Fonte: adaptado de CORREA et al. (2004 a, b, c).

Observam-se na figura 2 os ajustes da taxa de decomposição em relação à fitomassa inicial, apresentando-se curvas de tendência com padrões semelhantes entre a castanheira, o cupuaçuzeiro e a gliricídia, com uma fase de decomposição da fitomassa mais rápida, entre dez a vinte dias, seguida de um período mais lento. 


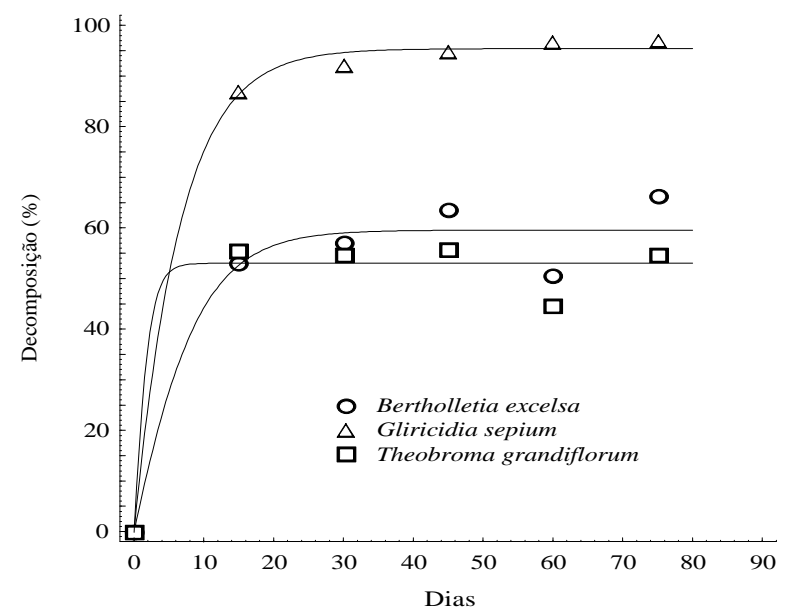

Figura 2. Taxa de decomposição de folhas de Bertholletia excelsa, Gliricidia sepium e Theobroma grandiflorum.

Figure 2. Decomposition rate of leaves of Bertholletia excelsa, Gliricidia sepium e Theobroma grandiflorum.

Fonte: adaptado de Correa et al. (2004 a, b, c).

Os diferentes tempos de decomposição entre as espécies avaliadas ocorreram devido às diferenças na composição bioquímica da biomassa. Compostos orgânicos de alta qualidade, ou seja, que se decompõem rapidamente, apresentam alto conteúdo de $\mathrm{N}$ e baixo conteúdo de lignina e de polifenóis. No caso de resíduos orgânicos de baixa qualidade, os níveis de $\mathrm{N}$ são baixos e os conteúdos de lignina e de polifenóis são altos (NYAMAI, 1992; YOUNG, 1990).

Entretanto, o aporte de biomassa de espécies com rápida e lenta taxa de decomposição, como por exemplo Gliricidia sepium e Inga edulis, respectivamente, possibilita sincronizar a liberação de nutrientes da biomassa para as culturas de interesse no período desejado, de acordo com os requerimentos de cada espécie (MAFONGOYA; GILLER; PALM, 1998).

\section{Produtividade das culturas anuais como componentes dos sistemas agroflorestais}

A tabela 5 apresenta a produtividade das culturas anuais nos sistemas agroflorestais $\mathrm{M}_{1}$ e $\mathrm{M}_{2}$ cultivadas nos três primeiros anos de implantação dos SAFs.

Tabela 5. Produtividade $\left(\mathrm{kg} \cdot \mathrm{ha}^{-1}\right)$ das culturas anuais nos SAFs.

Table 5. Annual crops productivity $\left(\mathrm{kg}^{-h^{-1}}\right)$ in agroforestry systems.

\begin{tabular}{lcccc}
\hline \multirow{2}{*}{ Componentes } & SAF & \multicolumn{3}{c}{ Ano } \\
\cline { 3 - 5 } & & $\mathbf{1}$ & $\mathbf{2}$ & $\mathbf{3}$ \\
\hline Arroz & $\mathrm{M}_{1}$ & 1550 & 1413 & $*$ \\
Milho & $\mathrm{M}_{2}$ & 3319 & $*$ & $*$ \\
Soja & $\mathrm{M}_{2}$ & $*$ & 1779 & $*$ \\
Mandioca & $\mathrm{M}_{1}$ & $*$ & $*$ & 4935 \\
& $\mathrm{M}_{2}$ & $*$ & $*$ & 10550 \\
\hline
\end{tabular}

*: não houve plantio.

Fonte: autores.

Em $\mathrm{M}_{1}$, o arroz apresentou baixa produtividade, variando de $1.550 \mathrm{~kg} \cdot \mathrm{ha}^{-1}$ a $1.413 \mathrm{~kg} \cdot \mathrm{ha}^{-1}$, no primeiro e no segundo ano, respectivamente. Esperava-se uma produtividade próxima à média regional, de aproximadamente $3.500 \mathrm{~kg} \cdot \mathrm{ha}^{-1}$ (CORDEIRO, 2002; CORDEIRO et al., 2003). Entretanto, a baixa fertilidade do solo nos dois primeiros anos de implantação dos SAFs limitou o potencial produtivo da cultura do arroz. A baixa fertilidade também afetou a produtividade da mandioca no modelo $\mathrm{M}_{1}$ (4.935 
$\mathrm{kg} . \mathrm{ha}^{-1}$ ), produzindo $47 \%$ a menos que no $\mathrm{M}_{2}$ e ficando abaixo da produtividade média regional, que é em torno de $13.000 \mathrm{~kg}^{-h^{-1}}$ (SCHWENGBER; SMIDERLE; MATTIONE, 2005).

No sistema $\mathrm{M}_{2}$, a produtividade obtida com o cultivo do milho foi de $3.319 \mathrm{~kg} \cdot \mathrm{ha}^{-1}$, com o da soja de 1.779 kg.ha ${ }^{-1}$ e com o da mandioca de $10.550 \mathrm{~kg}^{-} \mathrm{ha}^{-1}$, no primeiro, segundo e terceiro anos, respectivamente (Tabela 4).

Das culturas anuais plantadas tanto no $\mathrm{SAF} \mathrm{M}_{1}$ como no $\mathrm{SAF} \mathrm{M}_{2}$, a produtividade obtida com o cultivo da mandioca $\left(10.555 \mathrm{~kg} \cdot \mathrm{ha}^{-1}\right)$ foi ligeiramente inferior à média regional, que gira em torno de $13.000 \mathrm{~kg} \cdot \mathrm{ha}^{-1}$ (SCHWENGBER; SMIDERLE; MATTIONE, 2005). Esse fato contrariou as expectativas iniciais da equipe de pesquisa, que esperava um melhor rendimento das culturas do milho e da soja em relação à da mandioca. Entretanto, os fatores edafoclimáticos adversos àquelas culturas não prejudicaram o desenvolvimento da espécie menos exigente, a mandioca.

Esses resultados confirmam os obtidos por Santos; Rodriguez; Wandelli (2002), que avaliaram quatro modelos agroflorestais em áreas degradadas a $54 \mathrm{~km}$ de Manaus, nos quais a mandioca foi a cultura agrícola com a melhor produtividade quando comparada à do arroz e à do milho.

\section{Produtividade da bananeira (frutífera semiperene) nos sistemas agroflorestais}

Observa-se, na tabela 6, a produtividade da bananeira, plantada com uma densidade de 416 plantas.ha ${ }^{-1}$, nos modelos agroflorestais, avaliada durante cinco anos no SAF $M_{1}$ e durante seis anos no $\mathrm{M}_{2}$.

Tabela 6. Produtividade $\left(\mathrm{kg} \cdot \mathrm{ha}^{-1}\right)$ da bananeira em diferentes modelos agroflorestais.

Table 6. Productivity $\left(\mathrm{kg} \cdot \mathrm{ha}^{-1}\right)$ of banana in agroforestry systems.

\begin{tabular}{lcccccccc}
\hline \multirow{2}{*}{ Componente } & SAF & \multicolumn{10}{c}{ Ano } \\
\cline { 3 - 9 } & & $\mathbf{1}$ & $\mathbf{2}$ & $\mathbf{3}$ & $\mathbf{4}$ & $\mathbf{5}$ & $\mathbf{6}$ & $\mathbf{7}$ \\
\hline Bananeira & $\mathrm{M}_{1}$ & $*$ & 165 & 356 & 247 & 291 & 285 & $* *$ \\
& $\mathrm{M}_{2}$ & $*$ & 250 & 1407 & 276 & 1765 & 1104 & 732 \\
\hline
\end{tabular}

*: não houve plantio; **: foi naturalmente suprimida

Fonte: autores.

A fertilização complementar realizada nas bananeiras nos modelos $M_{1}$ e $M_{2}$, de 500 g.planta ${ }^{-1}$ de N-P-K na formulação 10-26-26 no ano três e de $210 \mathrm{~g}$ de sulfato de amônio, $900 \mathrm{~g}$ de superfosfato simples e $300 \mathrm{~g}$ de cloreto de potássio no ano quatro, não foi suficiente para aumentar satisfatoriamente a produtividade no modelo $\mathrm{M}_{1}$.

No início da fase produtiva (ano 2), sua produtividade foi a menor do período, $165 \mathrm{~kg} \cdot \mathrm{ha}^{-1}$. No terceiro ano, período que recebeu a fertilização complementar, a bananeira produziu $356 \mathrm{~kg}^{-h^{-1}}{ }^{-1}$, e nos anos seguintes a produtividade oscilou entre $247 \mathrm{~kg} \mathrm{ha}^{-1}$ e $291 \mathrm{~kg} \cdot \mathrm{ha}^{-1}$, valores muito inferiores aos obtidos no modelo $\mathrm{M}_{2}$.

$\mathrm{O}$ baixo rendimento da bananeira observado no $\mathrm{SAF} \mathrm{M}_{1}$ refletiu as características de baixa fertilidade química do solo. Os valores de $\mathrm{pH}$ se situaram entre 4.0 e 4.6, e os macronutrientes com os valores mais críticos foram o fósforo $\left(1 \mathrm{a} 5 \mathrm{mg} / \mathrm{dm}^{3}\right)$, o cálcio $\left(0.09\right.$ a $\left.0.44 \mathrm{cmolc} / \mathrm{dm}^{3}\right)$ e o magnésio $(0.1$ cmolc/ $\mathrm{dm}^{3}$ ).

Observando-se os resultados da bananeira no $\mathrm{SAF} \mathrm{M}_{2}$, pode-se verificar que a cultura respondeu à aplicação de insumos, produzindo de quatro a seis vezes mais quando comparada ao $\mathrm{SAF}_{1} \mathrm{M}_{1}$ nos anos três, cinco e seis, alcançando produtividade de 1.407 kg.ha ${ }^{-1}, 1.765$ kg.ha ${ }^{-1}$ e 1.104 kg.ha ${ }^{-1}$, respectivamente.

Os resultados da produtividade da bananeira alcançados no modelo $\mathrm{M}_{2}$ estão diretamente relacionados às condições edáficas, em que o pH aumentou de 4.5 a 5.8 e os valores mínimos de alumínio $\left(0.1 \mathrm{cmolc} / \mathrm{dm}^{3}\right)$ foram alcançados nos anos três e quatro. Dos macronutrientes, o fósforo apresentou o mais alto teor no ano três $\left(9.65 \mathrm{mg} / \mathrm{dm}^{3}\right)$, o cálcio oscilou seus valores entre 1.62 e $2.68 \mathrm{cmolc} / \mathrm{dm}^{3}$ e o magnésio variou entre 0.42 e $1.06 \mathrm{cmolc} / \mathrm{dm}^{3}$.

Gama (2003), estudando três arranjos de modelos agroflorestais compostos de castanheira, freijó, cupuaçuzeiro, pupunheira, pimenteira e bananeira, em Machadinho d'Oeste, Rondônia, avaliou a bananeira, com uma densidade de 278 plantas.ha ${ }^{-1}$, durante quatro anos. Seus resultados de produtividade foram bastante variados para os sistemas $\mathrm{T}_{2}$ (SAF composto por freijó, bananeira, pimenta-do-reino e cupuaçuzeiro) e T3 (SAF composto por pupunheira, bananeira, pimenta-do-reino e cupuaçuzeiro), iniciando com valores entre $2.110,78 \mathrm{~kg} \cdot \mathrm{ha}^{-1}$ e $2.536,75 \mathrm{~kg}^{-h^{-1}}$ no ano 1 , respectivamente, diminuindo para $301,28 \mathrm{~kg} \cdot \mathrm{ha}^{-1}$ e $539,95 \mathrm{~kg} \cdot \mathrm{ha}^{-1}$ no quarto ano de avaliação. Tais resultados ocorreram, de acordo 
com o autor, provavelmente devido a variações climáticas, diminuição da fertilidade do solo ou maior incidência de pragas.

\section{Produtividade do cupuaçuzeiro e da pupunheira (frutíferas perenes) nos diferentes modelos agroflorestais}

Os valores da produtividade do cupuaçuzeiro e da pupunheira nos sistemas $\mathrm{M}_{1}$ e $\mathrm{M}_{2}$ podem ser observados na tabela 7 .

Tabela 7. Produtividade do cupuaçuzeiro e da pupunheira nos SAFs.

Table 7. Cupuassu and Palm heart productivity in agroforestry systems.

\begin{tabular}{|c|c|c|c|c|c|c|c|c|}
\hline \multirow{2}{*}{ Componentes } & \multirow{2}{*}{ SAF } & \multicolumn{7}{|c|}{ Ano } \\
\hline & & 1 & 2 & 3 & 4 & 5 & 6 & 7 \\
\hline \multirow[t]{2}{*}{ Cupuaçuzeiro $\left(\mathrm{kg} \cdot \mathrm{ha}^{-1}\right)$} & $\mathrm{M}_{1}$ & + & + & + & + & 6 & 272 & 314 \\
\hline & $\mathrm{M}_{2}$ & + & + & + & + & 39 & 479 & 510 \\
\hline \multirow[t]{2}{*}{ Pupunheira (cachos.ha ${ }^{-1}$ ) } & $\mathrm{M}_{1}$ & $*$ & + & + & 35 & 43 & 62 & 75 \\
\hline & $\mathrm{M}_{2}$ & $*$ & + & + & 53 & 74 & 80 & 93 \\
\hline
\end{tabular}

*: não houve plantio; +: período de maturidade da cultura.

Fonte: autores.

O cupuaçuzeiro, plantado com uma densidade de 416 plantas.ha $^{-1}$, produziu frutos a partir dos cinco anos de idade. Seu melhor resultado ocorreu aos sete anos, com uma produtividade de $314 \mathrm{~kg}^{-h^{-1}} \mathrm{e}$ $510 \mathrm{~kg} . \mathrm{ha}^{-1}$ nos modelos $\mathrm{M}_{1}$ e $\mathrm{M}_{2}$, respectivamente. Esses resultados mostram que a produtividade no sistema $\mathrm{M}_{2}$ foi $62 \%$ maior quando comparada ao sistema $\mathrm{M}_{1}$. Pode-se atribuir o aumento da produtividade ocorrido entre os sistemas, em parte, ao manejo cultural adotado no $\mathrm{SAF} \mathrm{M}_{2}$.

O cupuaçuzeiro é uma espécie importante na composição dos sistemas agroflorestais estudados, presente na maior parte dos modelos desenhados por produtores rurais do estado de Roraima e da região amazônica como um todo (SÁ et al., 2000; SANTOS, RODRIGUEZ e WANDELLI, 2002; MENDES, 2002 e 2003).

A pupunheira, plantada na densidade de 338 plantas.ha $^{-1}$, iniciou sua produção de cachos aos três anos de idade. Nessa idade, o SAF $\mathrm{M}_{1}$ produziu 35 cachos.ha $^{-1}$ e o SAF $\mathrm{M}_{2} 53$ cachos.ha ${ }^{-1}$. Os melhores resultados ocorreram aos sete anos, com uma produtividade de 75 cachos.ha $^{-1}$ e 93 cachos.ha $^{-1}$ nos modelos $\mathrm{M}_{1}$ e $\mathrm{M}_{2}$, respectivamente, representando uma produtividade $24 \%$ maior no sistema $\mathrm{M}_{2}$. No caso das pupunheiras, a diferença na produtividade não foi tão evidente como o ocorrido na cultura do cupuaçuzeiro, apresentando uma resposta um pouco superior ao melhor manejo cultural no SAF $\mathrm{M}_{2}$.

Aos oito anos de idade, cada cupuaçuzeiro do $\mathrm{SAF} \mathrm{M}_{1}$ produzia de um a dois frutos, com peso médio de $808 \mathrm{~g}$. Nessa fase de produção, as plantas do SAF $\mathrm{M}_{1}$ produziram $380 \mathrm{~kg} \cdot \mathrm{ha}^{-1}$ de frutos, estabilizando a produção aos onze anos, com aproximadamente $660 \mathrm{~kg} \cdot \mathrm{ha}^{-1}$ (ARCO-VERDE; MOUR ̃̃O JÚNIOR, 2004a; ARCOO-VERDE; MOURÃO JÚNIOR, 2003b).

No SAF $\mathrm{M}_{2}$, constatou-se que cada planta de cupuaçuzeiro produziu uma média de dois frutos, com peso médio de $731 \mathrm{~g}$, produzindo $620 \mathrm{~kg} \cdot \mathrm{ha}^{-1}$ de frutos, atingindo a estabilidade da produção aos onze anos, com aproximadamente $830 \mathrm{~kg} \cdot \mathrm{ha}^{-1}$ (ARCO-VERDE; MOURÃO JÚNIOR, 2004a; ARCOVERDE; MOURÃO JÚNIOR, 2003b).

Valores semelhantes de produção de frutos aos oito anos $\left(2,3\right.$ frutos.planta $\left.^{-1} ; \mathrm{p}<0,30\right)$ foram obtidos por Marques; Ferreira; Carvalho (2001) em modelos de sistemas agroflorestais avaliados na região do Tapajós, Pará, com uma densidade de 136 plantas.ha $^{-1}$.

Conforme Arco-Verde;Mourão Júnior (2004a), a produção média dos frutos de cupuaçu é associada à precipitação semanal, sendo apresentados dois picos de produção. $\mathrm{O}$ primeiro ocorreu na semana final do mês de junho até a penúltima semana do mês de julho, representando o início de redução de chuvas (131-51 mm.semana ${ }^{-1}$ ) e assinalando $5 \%$ da produção total no sistema $\mathrm{M}_{2}$ e $37 \%$ da produção total no sistema $\mathrm{M}_{1}$. O segundo pico iniciou entre a segunda e a última semana de outubro, representando um período longo de estiagem ( 0 mm.semana $\left.{ }^{-1}\right)$, assinalando $93 \%$ da produção total no sistema $\mathrm{M}_{2}$ e $59 \%$ da produção total no sistema $\mathrm{M}_{1}$.

Devido ao período de maior produção de cupuaçu se situar no mês de outubro, ele pode ser comercializado tanto no mercado de Boa Vista, grande consumidor da produção do estado, quanto em Manaus, com safra nos meses de janeiro a maio (SOUZA et al., 1998), potencial mercado para o consumo de produtos agroflorestais procedentes do estado de Roraima. Essa diferença nos períodos de produção se 
deve às diferenças climáticas entre as duas cidades: Boa Vista está localizada no hemisfério Norte e Manaus ao sul da linha do Equador.

De acordo com Arco-Verde e Mourão Júnior (2004b), o início da coleta de cachos de pupunha no modelo $\mathrm{M}_{1}$ ocorreu na segunda quinzena do mês de julho (76 mm.semana ${ }^{-1}$ ), enquanto que no modelo $\mathrm{M}_{2}$ deu-se na segunda quinzena de agosto $\left(64 \mathrm{~mm} \cdot \mathrm{semana}^{-1}\right)$, com uma duração do ciclo de colheita de aproximadamente 70 dias no modelo $\mathrm{M}_{1}$ e de 40 dias no modelo $\mathrm{M}_{2}$.

$\mathrm{O}$ pico de produção dos modelos $\left(\mathrm{M}_{1}=70 \%\right.$ e $\mathrm{M}_{2}=80 \%$ da produção total) foi assinalado no período que compreende a segunda quinzena do mês de agosto (64 mm.semana $\left.{ }^{-1}\right)$. Já uma nova safra de cachos $(19 \%)$, no modelo $\mathbf{M}_{2}$, foi assinalada na primeira quinzena de setembro (98 mm.semana ${ }^{-1}$ ), enquanto que no modelo $M_{1}$ essa nova safra de cachos $(14 \%)$ foi assinalada na segunda quinzena de setembro (12 mm.semana ${ }^{-1}$ ) (ARCO-VERDE; MOURÃO JÚNIOR, 2003a).

Considerando a época de frutificação para a Amazônia Central, nos meses de dezembro a março (CLEMENT, 1999), tem-se que a pupunheira, como produto agroflorestal, tem potencial atrativo para o mercado interno, de Boa Vista, ou externo, como o de Manaus.

\section{Crescimento das espécies florestais}

Os resultados do crescimento da castanha-do-brasil e da cupiúba, plantadas nos modelos $\mathrm{M}_{1}$ e $\mathrm{M}_{2}$ e em monocultivo, são apresentados na tabela 8 .

A castanheira apresentou um crescimento em DAP e em altura total de $10,5 \mathrm{~cm}$ e $6,1 \mathrm{~m}$ no sistema $\mathrm{M}_{1}$ e 4,9 cm e 3,6 m no sistema $\mathrm{M}_{2}$, respectivamente. Essa diferença entre os modelos representa um maior crescimento do sistema $\mathbf{M}_{1}$ quando comparado ao sistema $\mathbf{M}_{2}(114 \%$ em DAP e $69 \%$ em altura), resultado que pôde ser observado em todas as repetições desde a instalação do experimento.

Tabela 8. Crescimento da castanheira e da cupiúba em sistemas agroflorestais e em monocultivo. Table 8. Brazil nut and cupiúba growth in agroforestry systems and monoculture.

\begin{tabular}{|c|c|c|c|c|c|c|}
\hline \multirow{2}{*}{ Espécies } & \multirow{2}{*}{ Sistema } & \multirow{2}{*}{$\mathbf{N}$} & \multirow{2}{*}{ Idade (anos) } & \multicolumn{3}{|c|}{ Parâmetros } \\
\hline & & & & DAP (cm) & Altura (m) & Volume $\left(\mathrm{m}^{3}\right)$ \\
\hline \multirow[t]{3}{*}{ Castanheira } & $\mathrm{M}_{1}$ & 52 & 4 & 10,5 & 6,1 & 1,4111 \\
\hline & $\mathrm{M}_{2}$ & 52 & 4 & 4,9 & 3,6 & 0,3536 \\
\hline & Mono & 52 & 7 & 13,5 & 11,3 & 3,9196 \\
\hline \multirow[t]{3}{*}{ Cupiúba } & $\mathrm{M}_{1}$ & 52 & 5 & 5,3 & 4,3 & 0,4155 \\
\hline & $\mathrm{M}_{2}$ & 52 & 5 & 5,1 & 4,9 & 0,4276 \\
\hline & Mono & 52 & 7 & 9,7 & 8,5 & 1,6407 \\
\hline
\end{tabular}

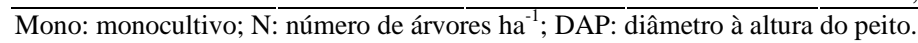

Equação de volume utilizada: $v=b_{o}+b_{1} d^{2} h$ (Spurr) (TONINI; ARCO-VERDE; SÁ, 2005).

Fonte: autores.

Esperava-se maior crescimento da castanheira no sistema $\mathrm{M}_{2}$, onde a gradagem e a correção de solo deveriam fornecer melhores condições para o desenvolvimento da espécie. Entretanto, a castanheira se mostrou mais adaptada às condições de baixa fertilidade e/ou menor competitividade diante de outras espécies do sistema.

Em relação à cupiúba, os resultados de crescimento em DAP e altura foram semelhantes em ambos os sistemas, com DAP ligeiramente superior $(0,2 \mathrm{~cm})$ no sistema $\mathrm{M}_{1}$. Entretanto, os incrementos, tanto em altura como em diâmetro, para os dois SAFs avaliados, ficaram muito aquém dos resultados obtidos para os plantios solteiros, que produziram em torno de $80 \%$ a mais que os sistemas agroflorestais.

Verificou-se que as espécies florestais cresceram anualmente, em média, cerca de $1 \mathrm{~cm}$ em DAP e $1 \mathrm{~m}$ em altura, exceto para o DAP da castanheira no sistema $\mathrm{M}_{1}$, no qual alcançou $2,1 \mathrm{~cm} . \mathrm{ano}^{-1}$.

\section{CONCLUSÕES E RECOMENDAÇÕES}

- Os componentes agroflorestais obtiveram melhores resultados de crescimento e produtividade no modelo agroflorestal que foi gradeado e melhor fertilizado.

- A biomassa proveniente das podas de Inga edulis e Gliricidia sepium contribuiu para a manutenção da fertilidade do solo nos SAFs, principalmente com o aporte de N, Ca e Mg. 
- O aporte de biomassa de espécies com rápida e lenta taxa de decomposição, como Gliricidia sepium e Inga edulis, respectivamente, possibilita sincronizar a liberação de nutrientes da biomassa para as culturas no período desejado, de acordo com os requerimentos de cada espécie.

- O plantio de espécies adubadoras é importante em solos de baixa fertilidade.

- A mandioca é uma cultura que merece especial atenção, particularmente para o uso em SAFs, tendo em vista tanto sua produtividade como sua adotabilidade no meio rural amazônico.

- O cupuaçuzeiro foi a espécie que apresentou melhor resposta produtiva em solos com maior fertilidade, o que a qualifica como atrativa para compor sistemas agroflorestais na Amazônia.

- As espécies florestais avaliadas apresentaram crescimento moderado nos SAFs.

- Deve ser reavaliada a fertilização utilizada em relação à matéria orgânica e potássio, uma vez que esses elementos não aumentaram seus teores durante os cinco anos de estudo.

\section{REFERÊNCIAS}

ARCO-VERDE, M. F.; MOURÃO JÚNIOR, M. Época de produção da pupunha (Bactris gasipaes) como componente de sistemas agroflorestais em Roraima. Comunicado Técnico, Boa Vista, RR, n. 19, 5 p., 2003a.

ARCO-VERDE, M. F.; MOURÃO JÚNIOR, M. Produção de cupuaçu (Theobroma grandiflorum) como componente de sistemas agroflorestais em Roraima. Comunicado Técnico, Boa Vista, n. 16, 5 p., 2003 b.

ARCO-VERDE, M. F.; MOURÃO JUNIOR., M. Ciclo de produção do cupuaçuzeiro como componente de sistemas agroflorestais em Roraima. In: CONGRESSO BRASILEIRO DE SISTEMAS AGROFLORESTAIS: SAFs: Desenvolvimento com Proteção Ambienta, 5., 2004, Curitiba. Anais... Colombo: Embrapa Florestas, 2004a. p. 488-490.

ARCO-VERDE, M. F.; MOURÃO JUNIOR., M. Ciclo de produção da pupunheira como componente de sistemas agroflorestais em Roraima. In: Congresso Brasileiro de Sistemas Agroflorestais: SAFs: Desenvolvimento com Proteção Ambiental. Anais... Curitiba. Embrapa Florestas, 2004b. p. 491-493.

CLEMENT, C. R. 1999. Introdução à pupunha. Pupunha-Net. Disponível em: <www.inpa.gov.br/pupunha/artigos/crc1.html>. Acesso em: 12/04/2004.

CORDEIRO, A. C. C. Linhagens promissoras de arroz de terras altas para Roraima no período de 1997 a 2001. In: CONGRESSO DA CADEIA PRODUTIVA DO ARROZ, 1.; REUNIÃO NACIONAL DE PESQUISA DE ARROZ - RENAPA, 7, 2002, São Paulo. Anais... Santo Antônio de Goiás: Embrapa Arroz e Feijão, 2002. v. 1, p.192-193. (Documentos, n. 134).

CORDEIRO, A. C. C.; MEDEIROS, R. D. de; PEREIRA, P. R. V. da S.; MOREIRA, M. A. B. Orientações técnicas para o cultivo do arroz de terras altas em Roraima. Circular Técnica, Boa Vista, Roraima, n. 1, 21 p., 2003.

CORREA, E. F.; ARCO-VERDE, M. F.; MOURÃO JUNIOR., M.; LIMA, H. N. Decomposição de folhas de castanha-do-Brasil (Bertholletia excelsa, Lecythidaceae) em um modelo de sistema agroflorestal de Roraima. In: CONGRESSO BRASILEIRO DE SISTEMAS AGROFLORESTAIS: SAFs: Desenvolvimento com Proteção Ambiental, 5., 2004, Curitiba. Anais... Colombo: Embrapa Florestas, 2004a. p. 67-69.

CORREA, E. F.; ARCO-VERDE, M. F.; MOURÃO JUNIOR., M.; LIMA, H. N. Decomposição de folhas de cupuaçuzeiro (Theobroma grandifolium, Sterculiaceae) em um modelo de sistema agroflorestal de Roraima. In: CONGRESSO BRASILEIRO DE SISTEMAS AGROFLORESTAIS: SAFs: Desenvolvimento com Proteção Ambiental, 5., 2004, Curitiba. Anais... Colombo: Embrapa Florestas, 2004b. p. 70-72.

CORREA, E. F.; ARCO-VERDE, M. F.; MOURÃO JUNIOR., M.; LIMA, H. N. Decomposição de folhas de gliricídia (Gliricidia sepium, Leg. Papilionoidea) em um modelo de sistema agroflorestal de Roraima. In: CONGRESSO BRASILEIRO DE SISTEMAS AGROFLORESTAIS: SAFs: Desenvolvimento com Proteção Ambiental, 5., 2004, Curitiba. Anais... Colombo: Embrapa Florestas, 2004. p. 73-75. 
EMBRAPA. Sistema Brasileiro de classificação de solos. Brasília, DF: CNPS, 1999. 412p.

FASSBENDER, H. W. Modelos edafológicos de sistemas agroforestales. Turrialba: CATIE, $491 \mathrm{p}$. 1993. (Serie de materiales de enseñanza no. 29).

FASSBENDER, H. W.; BORNEMISZA, E. Química de suelos con énfasis en suelos de América Latina. San José, Costa Rica: IICA, 1987. 420 p.

GAMA, M. M. B. Análise técnica e econômica de sistemas agroflorestais em Machadinho d'Oeste, Rondônia. 112 f. Tese (Doutorado em Ciência Florestal) - Universidade Federal de Viçosa, Viçosa, MG, 2003.

KASS. D. C. L. Fertilidad de Suelos. San José, Costa Rica: EUNED, 1996. 272 p.

KRISHNAMURTHY, L.; ÁVILA, M. Agroforestería Básica. México, D.F: Programa de las Naciones Unidas para el Medio Ambiente - PNUMA. 1999. 340 p. (Série Textos Básicos para la Formación Ambiental, n. 3).

MAFONGOYA, P. L. Multipurpose tree pruning as a source of nitrogen to maize (Zea mays L.) under semiarid conditions in Zimbabwe. 140 p. Tese (Doutorado) - Universidade da Florida, USA. 1995 .

MAFONGOYA, P. L.; GILLER, K. E.; PALM, C. A. Decomposition and nitrogen release patterns and tree pruning and litter. Agroforestry Systems, Dordrecht, n. 38, p. 77-97, 1998.

MARQUES, L. C. T.; FERREIRA, C. A. P.; CARVALHO, E. J. M. Sistema agroflorestal em área de pequeno produtor na região do Tapajós, estado do Pará - Avaliação após doze anos de implantado. Belém: Embrapa Amazônia Oriental, 2001. 19 p. (Documentos, n. 99).

MENDES, F. A. T. Avaliação de modelos de SAFs em pequenas propriedades selecionadas no município de Tomé-Açú, Estado do Pará. In: CONGRESSO BRASILEIRO DE SISTEMAS AGROFLORESTAIS, 4., 2002, Ilhéus, Bahia. Anais do... Ilhéus: CEPLAC, 2002. 3 p.

MENDES, F. A. T. Avaliação de modelos simulados de sistemas agroflorestais em pequenas propriedades cacaueiras selecionadas no município de Tomé-Açú, no Estado do Pará. Informe Gepec, Toledo, v. 07, n. 1, p. 118 - 144, 2003.

MOURÃO JUNIOR., M.; XAUD, M. R.; XAUD, H. A. M.; MOURA NETO, M. A. de; ARCO-VERDE, M. F.; PEREIRA, P. R. V. S.; TONINI, H. Precipitação pluviométrica em áreas de transição savana-mata de Roraima: campos experimentais Serra da Prata e Confiança. Comunicado Técnico, Boa Vista, n. 17. 7 p., 2003.

NAIR, P. K. R. An Introduction to Agroforestry. Netherlands: Kluwer Academic, 1993. 499 p.

NYAMAI, D. O. Investigations on decomposition of foliage of woody species using a perfusion method. Plant and Soil, The Hague, n. 139, p. 239 - 245, 1992.

SÁ, C. P. de; SANTOS, J. C. dos; LUNZ, A. M. P.; FRANKE, I. L. Análise financeira e institucional de três principais sistemas agroflorestais adotados pelos produtores do Reca. Circular Técnica, Rio Branco, AC, n. 33, 12 p. 2000.

SANTOS, M. J. C.; RODRIGUEZ, L. C. E.; WANDELLI, E. V. Avaliação econômica de quatro modelos agroflorestais em áreas degradadas por pastagens na Amazônia Ocidental. Scientia Forestales, Piracicaba, n. 62, p. 48-61, 2002.

SCHWENGBER, D. R.; SMIDERLE, O. J.; MATTIONI, J. A. M. Mandioca: recomendações para o plantio em Roraima. Circular Técnica, Boa Vista, n. 5, 30 p., 2005.

SOUZA, A. G. C.; SILVA, S. E. L.; TINOCO, P. B.; GUIMARÃES, R. R.; SÁ SOBRINHO, A. F. Cadeia produtiva do cupuaçu no Amazonas. Documentos, Manaus, n. 17, 1998.

YOUNG, A. Agroforestry for Soil Management. Wallingford, Oxon: CAB International, 1998. 320 p. 